LAWRENCE LIVERMORE NAT IO N A L LABORATORY

\title{
Thermally induced dephasing in periodically poled KTiOPO4 nonlinear crystals
}

Z. M. Liao, S. A. Payne, J. W. Dawson, A. D. Drobshoff, C. A. Ebbers, D. M. Pennington, I. Jovanovic, L. R. Taylor

March 24, 2004

Nonlinear Optics: Materials, Fundamentals, and Applications Waikoloa, $\mathrm{HI}$, United States August 2, 2004 through August 6, 2004 
This document was prepared as an account of work sponsored by an agency of the United States Government. Neither the United States Government nor the University of California nor any of their employees, makes any warranty, express or implied, or assumes any legal liability or responsibility for the accuracy, completeness, or usefulness of any information, apparatus, product, or process disclosed, or represents that its use would not infringe privately owned rights. Reference herein to any specific commercial product, process, or service by trade name, trademark, manufacturer, or otherwise, does not necessarily constitute or imply its endorsement, recommendation, or favoring by the United States Government or the University of California. The views and opinions of authors expressed herein do not necessarily state or reflect those of the United States Government or the University of California, and shall not be used for advertising or product endorsement purposes. 


\title{
Thermally induced dephasing in periodically poled $\mathrm{KTiOPO}_{4}$ nonlinear crystals
}

\author{
Z. M. Liao, S. A. Payne, J. W, Dawson, A. D. Drobshoff, C. A. Ebbers, D. M. Pennington, and I. Jovanovic \\ Lawrence Livermore National Laboratory, 7000 East Avenue, L-482, Livermore, CA 94550 \\ liao2@llnl.gov \\ L. R. Taylor \\ European Southern Observatory, Garching, Germany D85748
}

\begin{abstract}
Experimental data that exhibits a continuous-wave, second-harmonic intensity threshold $\left(15 \mathrm{~kW} / \mathrm{cm}^{2}\right)$ that causes two-photon nonlinear absorption which leads to timedependent photochromic damage in periodically poled $\mathrm{KTiOPO}_{4}$ is presented and verified through a thermal dephasing model.

(a) 2004 Optical Society of America

OCIS codes: (190.0190) Nonlinear Optics, (190.2620) Frequency Conversion, (190.4400) Nonlinear Optics, Materials
\end{abstract}

\section{Introduction}

Periodically poled nonlinear materials have the potential to serve as versatile devices for laser developers because of their ability to quasi-phase-match (QPM) over long lengths of crystal and allow noncritical phase matching for tight focusing geometries, thereby enabling efficient frequency conversion of continuous-wave $(\mathrm{CW})$ and low-peakpower pulsed lasers. A number of crystals have been successfully poled for frequency conversion of which periodically poled $\mathrm{LiNbO}_{3}$ (PPLN) and $\mathrm{KTiOPO}_{4}$ _PPKTP) have produced the most success. PPLN has demonstrated high conversion efficiency $(2.7 \mathrm{~W}$ out of $6.5 \mathrm{~W})$ in $\mathrm{CW}$ single-pass operation before exhibiting photorefractive damage [1]. Recently, periodically poled stoichiometric $\mathrm{LiTaO}_{3}$ (PPSLT) has successfully demonstrated 300 minutes of $\mathrm{CW}$ single-pass second harmonic generation at 1064 nm (1.6 W out of 18_W) without observable damage [2]. Unfortunately, there have been no reported CW high-power studies using PPKTP, although no damage was observed in low power regime $(\sim \mathrm{mW})$ [3]. In pulsed systems however, PPKTP has been shown to be capable of generating large amounts of 532_nm light (up to 6_W average power) [4] without observing degradation.

In bulk KTP crystals, laser intensity induced damage (gray tracking) is well documented [5]. The transmission degradation of the crystal across the visible wavelengths is generally attributed to the creation of color centers through two-photon absorption. We describe our assessment of time-dependent conversion efficiency degradation in PPKTP in a $\mathrm{CW}_{2}$ single-pass configuration. This investigation has important consequences for pulsed operation as well, particularly for longer pulses and for high-average-power systems.

\section{Theory of nonlinear conversion with losses}

The exact treatment of second harmonic generation (SHG) consisted of the solving the coupled wave equation of the pump and the signal fields as it propagates through the crystal [6]. S everal works treated reduction of coupled wave equations to a close-form analytical solution, notably Boyd and Kleinman (BK) [7].

The solution of SHG in the BK focusing regime (tight focusing) is given as

$$
P_{2 \omega}=f g L \eta P_{1 \omega}^{2},
$$

where $f$ is the focusing factor that accounts for non-ideal focusing, $g$ is the nonlinear conversion coefficient, $\eta$ is the thermal dephasing factor averaged over the crystal length $\underline{L}$, and $P_{I \omega}$ and $P_{2 \omega}$ are the fundamental and the secondharmonic powers, respectively. The thermal dephasing factor $\eta$ is a function of the temperature detuning $(\Delta T)$ and is directly related to the absorption of the crystal. The total absorption of the crystal is then the sum of the linear absorption $\left(\alpha_{L}\right)$ due to impurities and the time-dependent nonlinear absorption due to precursor defects, $\alpha=\alpha_{L}+\alpha_{s}[1$ $\left.\exp \left(-\beta I^{2}{ }_{2 \omega} t\right)\right]$ where, $\beta$ is the defect-creation coefficient and $\alpha_{\underline{S}}$ is the saturated nonlinear absorption. Defect generation is a function of $I_{2 \underline{\omega}}^{2}$ because the process is a two-photon absorption interaction. A time-dependent, 
transcendental equation that takes into account the thermally induced dephasing from the temperature raise due to nonlinear absorption can be written as

$$
\begin{aligned}
P_{2 \omega}= & f g P_{1 \omega}{ }^{2} \cdot \operatorname{sinc}^{2}\left[\frac{\beta_{T} L}{2}\left\{\frac{1+2 \ln \left(a / w_{0}\right)}{4 \pi K}\left\{\alpha_{L}+\alpha_{s}\left(1-\exp \left(-\beta I_{2 \omega}{ }^{2} t\right)\right)\right\} P_{2 \omega}+\delta T\right\}\right] . \\
& \cdot\left\{\frac{1-\exp \left[-\left\{\alpha_{L}+\alpha_{s}\left(1-\exp \left(-\beta I_{2 \omega}{ }^{2} t\right)\right)\right\} L\right]}{\alpha_{L}+\alpha_{s}\left(1-\exp \left(-\beta I^{2}{ }^{2} t\right)\right.}\right\}
\end{aligned}
$$

The first factor accounts for the nonlinear conversion, and the second factor accounts for reduction of second harmonic light due to dephasing, where $\beta_{T}$ is the temperature-dependent phase mismatch, $a$ is the crystal width, $w_{0}$ is the focused spot size, and $K$ is the thermal conductivity. An alignment factor $(\delta T)$ that accounts for the initial nonoptimal QPM temperature setting is also added. The last factor, the effective crystal length, is adjusted for the loss of second-harmonic light due to absorption. The heating is actually a function of varying beam waist, which is treated here as a lumped parameter. This averaged dephasing model is therefore only valid when the dephasing is small enough throughout the crystal length to create only a perturbative dephasing.

\section{Experimental setup and results}

The experimental setup for nonlinear conversion testing is shown in Fig. 1. A single transverse mode $\left(\mathrm{M}^{2}=1.1\right)$ with correct polarization at $1064 \mathrm{~nm}$ is used as the pump. The Raicol (Israel) flux-grown PPKTP crystal has dimensions of $1 \times 2 \times 30 \mathrm{~mm}^{3}$ and a period of $9.1 \mu \mathrm{m}$ for a calculated phase-matched temperature of $148^{0} \mathrm{C}$ for SHG. Different lens systems were used to obtain various peak pump intensities to study the effects of confocal and strong focusing. The second harmonic power is separated from the residual pump power using two dichroic mirrors designed to pass the 1064-nm light and reflect the 532-nm light.

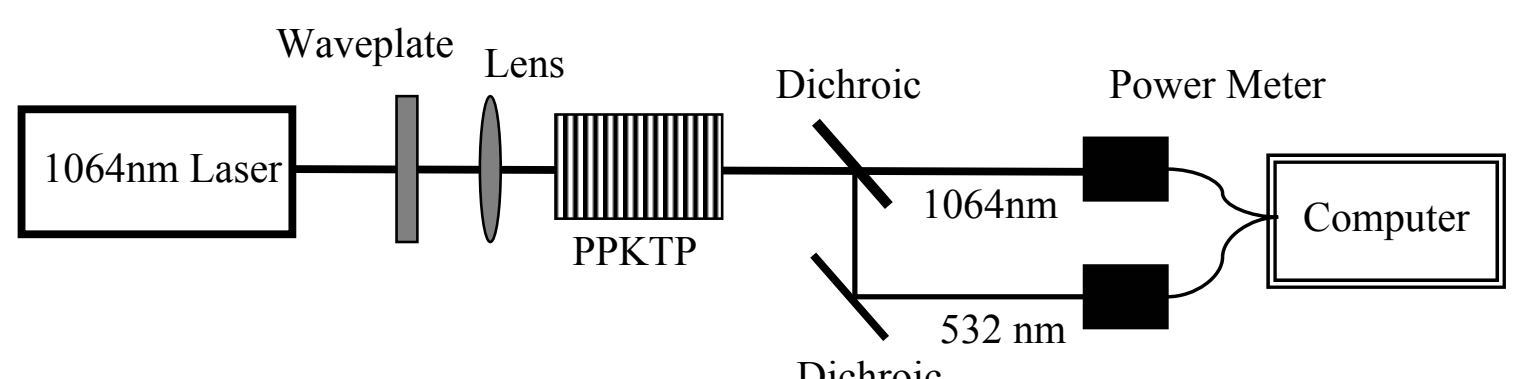

Dichroic

Fig. 1. Experimental setup

SHG power as a function of time for several levels of input power is displayed in Fig. 2. A threshold intensity of $90 \mathrm{~kW} / \mathrm{cm}^{2}(\sim 5 \mathrm{~W})$ is measured, below which no degradation is observed. The rate of SHG degradation increases with increased pump power, but the steady-state conversion power remains nearly constant. This indicates that a maximum precursor site concentration exists. The time characteristics of defect or color-center creation matches well with gray-tracking of bulk KTP that has undergone damage-protective treatment, as described by Murk et al. [5] The saturation time is on the order of 2 hours, but unlike the bulk KTP results [5], we were unable to produce any recovery after 2-3 days even after the crystal has been annealed at T 500K. Furthermore, when the damage is initiated, at pump intensity below the threshold level, there is no degradation in the conversion efficiency. In addition, conversion degradation only occurs above threshold intensity, indicating that the degradation is due to dephasing as opposed to actual physical damage. Theoretical predictions from our model are in good agreement with experiment data, as evident in Fig. 2. From our calculations, a defect creation rate of $\beta=3.75 \times 10^{3} \mu \mathrm{m}^{4} /\left(\mathrm{W}^{2} \mathrm{~s}\right)$ is obtained along with a saturated absorption of $14 \% / \mathrm{cm}$. The linear absorption coefficient by contrast is only $1 \% / \mathrm{cm}$. Furthermore, we were able to independently measure the QPM temperature of the crystal under various pump intensities and matched it with our model predictions. 


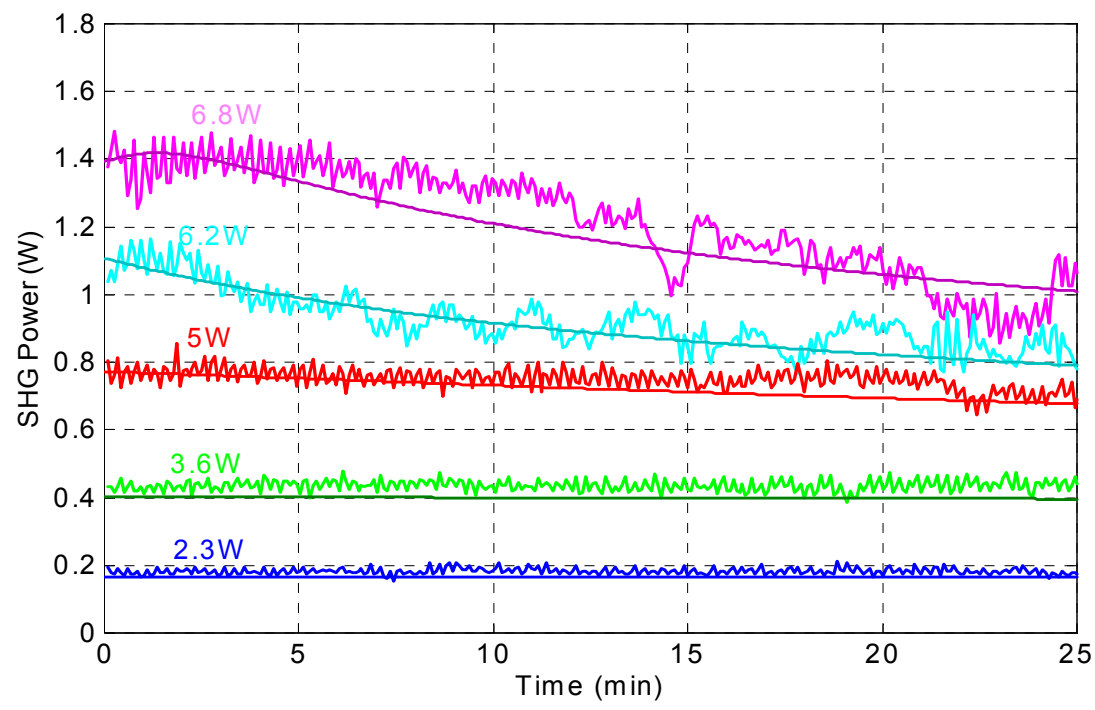

Fig. 2. Experimental (dotted) and theoretical (solid) SHG power vs. time for various input powers

\section{Conclusion}

We present experimental data on conversion efficiency degradation in a single-pass, CW SHG system using PPKTP. We also present in detail a successful model based on the spatial-temporal thermally induced dephasing through local heating. The generation of defect sites leads to nonlinear absorption that is found to be unrecoverable and is activated by a two-photon absorption of the $532-\mathrm{nm}$ light at a threshold power of $800 \mathrm{~mW}\left(15 \mathrm{~kW} / \mathrm{cm}^{2}\right)$. Our results indicate that PPKTP is limited in average power by thermal effects, and in peak intensity by nonlinear absorption.

Independent study of pulsed operation may provide a more accurate estimate of the crystal degradation by nonlinear absorption. The results of this study affect a number of other devices based on nonlinear frequency conversion, such as optical parametric amplifiers, optical parametric oscillators, and parametric generators. Future studies will be focused on peak and average power limitations of novel nonlinear periodically poled materials, such as PPSLT.

This work was done under the auspices of the U.S. Department of Energy by the University of California Lawrence Livermore National Laboratory under Contract W-7405-ENG-48 and funded through LLNL office of LDRD (01ERD-83 and 03-LW-040) and the NSF Center for Adaptive Optics (CFAO).

\section{References}

1. G. Miller, R. Batchko, W. Tulloch, D. Weise, M. Fejer, and R. Byer, "42\%-Efficient Single-Pass CW SecondHarmonic Generation in Periodically Poled Lithium Niobate", Opt. Lett. 22, 1834-1836 (1997).

2. M. Katz, R. Route, D. Hum, R. Roussev, K. Parameswaran, V. Kondilenko, G. Miller, and M. Fejer, "NearStoichiometric 1\% Mg-Doped $\mathrm{LiNbO}_{3}$ and Stoichiometric $\mathrm{LiTaO}_{3}$ Fabricated by Vapor Transport Equilibration for Frequency Conversion," in Stanford Photonic Research Center Annual Report (2003).

3. A. Arie, G. Rosenman, V. Mahal, A. Skliar, M. Oron, M. Katz, and D. Eger, "Green and Ultraviolet Quasi-

| Phase-Matched Second Harmonic Generation in Bulk Periodically-Poled KTiOPO4", Opt. Commun. 142, 265268 (1997).

4. S. V. Popov, S. V. Chernikov, and J. R. Taylor, "6-W Average Power Green Light Generation using Seeded

| High Power Ytterbium Fibre Amplifier and Periodically Poled KTP”, Opt. Commun. 174, 231-234 (2000).

5. V. Murk, V. Denks, A. Dudelzak, P. Proulx, and V. Vassiltsenko, "Gray Tracks in $\mathrm{KTiOPO}_{4}$ : $\mathrm{Mechanism}$ of Creation and Bleaching", Nuclear Instr. \& Meth. In Phys. Res. B, 141 472-476 (1998).

6. R. Boyd, in Nonlinear Optics, Academic Press, San Diego, 1992.

7. G. D. Boyd and D. A. Kleinman, "Parametic Interaction of Focused Gaussian Light Beams", J. Appl. Phy s. 39, 3597-3639 (1968). 